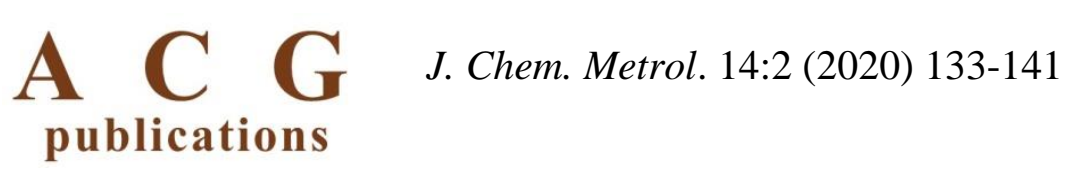

journal of chemical metrology

\title{
Oleuropein levels of Anatolian olive leaves and correlated antioxidant, antidiabetic, and anti-inflammatory activities
}

\author{
Zeynep Kalaycığlu@, Merve Kopar $\odot$ and F. Bedia Erim $\oplus^{*}$ \\ Istanbul Technical University, Department of Chemistry, Maslak, Istanbul, Türkiye
}

(Received October 10, 2020; Revised November 19, 2020; Accepted November 19, 2020)

\begin{abstract}
Oleuropein, the major active compound in olive leaf, has been of considerable interest for its many health benefits. This work aims the determination of oleuropein in olive leaves from Turkey by a rapid and simple capillary electrophoretic method, and then to correlate the oleuropein amounts and the bioactivities of olive leaf extracts. The optimal separation medium was composed of $30 \mathrm{mmol} / \mathrm{L}$ borate $(\mathrm{pH}: 9.6), 25 \mathrm{mmol} / \mathrm{L} 2$-hydroxypropyl- $\beta$ cyclodextrin (2-HP- $\beta-\mathrm{CD}), 10 \%$ (v/v) methanol. Moreover, olive leaf extracts were examined for antioxidant, antidiabetic, and anti-inflammatory activities. The antioxidant activities were determined by 1,1-diphenyl-2-picrylhydrazyl (DPPH) radical scavenging activity. The antidiabetic activities were predicted using $\alpha$-glucosidase inhibitory effects. For the anti-inflammatory activities of the extracts, their reduction power of pro-inflammatory tumor necrosis factor (TNF)-alpha were measured. Oleuropein concentration ranged between 11.7-106 mg/g dry leaf. Strong correlations were detected between each biological activity and the oleuropein content of olive leaves.
\end{abstract}

Keywords: Capillary electrophoresis; olive leaf; oleuropein; antioxidant activity; anti-inflammatory activity; antidiabetic activity. ( 2020 ACG Publications. All rights reserved.

\section{Introduction}

The olive tree (Olea europaea L.), native to the Mediterranean region, is famous for its fruit, olive. Olive has unique characteristics and also is the primary source of olive oil which has been known for its value for centuries [1]. Although olive is also widely cultivated in several parts of the world, the Mediterranean region is still the major production area. Turkey is one of the producing countries and is ranked as the world's second-biggest producer [2].

Recently, there has been an increasing scientific interest on the olive by-products such as olive leaves [3]. Olive leaf is considered as both an important agricultural biomass and an easily available industrial by-product. In ancient times, olive leaves have been used in the treatment of malaria and fever.

Olive leaf and olive leaf extracts have been used in pharmaceutical and food industries due to their many bioactivities $[4,5]$. Hence, the chemical composition of olive leaf has attracted a great deal of attention. The presence of phenolic derivatives, pentacyclic triterpenes, and alditols in significant concentrations is responsible for these various pharmacological properties.

Oleuropein is one of the most important phenolic derivatives present in the olive leaf. The pharmacological importance of oleuropein stands out with its antioxidant, anti-inflammatory,

*Corresponding author:E-Mail:erim@itu.edu.tr

The article was published by ACG Publications

http://www.acgpubs.org/journal/journal-of-chemical-metrologyJuly-December 2020 EISSN:1307-6183

DOI:http://doi.org/10.25135/jcm.51.20.04.1632 
antidiabetic, anticancer, antimicrobial, and antiviral activities [6-10].The scientific studies on thetherapeutic effects of oleuropein have been extended, and specific reviews were recently reported [11, 12].The typical bitterness of olive is derived from the oleuropein.

The level of oleuropein changes according to the olive varieties. Thus, the oleuropein amount is an indicator showing the quality of olive leaf.

Oleuropein in olive leaf extracts has been quantified by HPLC [13-15].However, capillary electrophoresis $(\mathrm{CE})$ presents rapid analysis time, high efficiency, and low sample consumption compared to HPLC. CE methods developed for the determination of oleuropein in olive products were reported [16, 17].

In the present study, we developed a CE method for the analysis of oleuropein in the olive leaf extracts. The optimized method was used to quantify oleuropein in 7 olive leaves collected from different regions of Anatolia and in 2 commercial olive leaf tea samples. Then, the antioxidant activity, antidiabetic activity, and anti-inflammatory activity of the extracts were evaluated. Finally, a statistical correlation was established between the oleuropein contents of leaves and their bioactivities. To our knowledge, this is the first capillary electrophoretic method applied for the oleuropein analysis in olive leaf. Moreover, the correlations between oleuropein contents of olive leaves from different parts of Anatolia and their antidiabetic and anti-inflammatory activities were searched in this study for the first time.

\section{Materials and Methods}

\subsection{Chemicals}

Standard oleuropein, 2-hydroxypropyl- $\beta$-cyclodextrin (average $\mathrm{M}_{\mathrm{w}} \sim 1460$ and 0.8 molar substitution), $\alpha$-glucosidase (EC 3.2.1.20), genistein, $p$-nitrophenyl- $\alpha$-D-glucopyranoside substrate ( $p$ NPG), and butylated hydroxyanisole (BHA) were obtained from Sigma (Steinheim, NRW, Germany). Disodium tetraborate decahydrate, sodium hydroxide, 1,1-diphenyl-2-picrylhydrazyl, sodium carbonate, methanol, sodium dihydrogen phosphate dihydrate, and disodium hydrogenphosphate dodecahydrate were from Merck (Darmstadt, Germany).

The stock solution of oleuropein was prepared by methanol at $1000 \mu \mathrm{g} / \mathrm{mL}$ concentration and kept at $4{ }^{\circ} \mathrm{C}$. Calibration solutions were prepared from this stock solution diluting with methanol.

\subsection{Plant Materials and Sample Preparation}

Olive growing generally spreads across the Aegean and Marmara Regions of Turkey, yielding a very rich heritage of native varieties. The mature green olive leaves were collected from different areas of Turkey, including Bursa, İstanbul, and Yalova from the Marmara Region; İzmir, Manisa, Balıkesir, and Muğla from the Aegean Region. The collection was carried out in the summer. Fresh leaves were airdried at room temperature for $72 \mathrm{~h}$. Dried leaves were ground in a laboratory blender. Two different brands of commercial olive leaf tea samples were supplied from markets in Istanbul.

Q1

The homogenized olive leaves were accurately weighed as $0.1 \mathrm{~g}$ and extracted with $5 \mathrm{~mL}$ of $80 \%$ methanol - 20\% water $(v / v)$ mixture. The mixture was vortexed for $5 \mathrm{~min}$ and then ultrasonicated for $1 \mathrm{~h}$. After centrifugation at $3500 \mathrm{rpm}$ for $10 \mathrm{~min}$, it was filtered through a filter paper. The sediments were extracted again with $5 \mathrm{~mL}$ of $80 \%$ methanol $-20 \%$ water $(v / v)$ for $30 \mathrm{~min}$ by ultrasonicator. Combined extracts were diluted to $10 \mathrm{~mL}$. Injections were done after the extracts passed through 0.45 micron filters.

\subsection{Instrumentation}

Agilent 1600 capillary electrophoresis (Waldbronn, Germany) was utilized for oleuropein analysis. The detection was done with a diode-array detector (DAD). Data were collected and processed by Agilent ChemStation software. Separations were performed in a fused silica capillary column $(50 \mathrm{~cm}$ length and $50 \mu \mathrm{m}$ diameter) purchased from Polymicro Technologies, Phoenix, AZ, USA. Injections were done at 50 mbar pressure for 6 seconds. The temperature was fixed at $25^{\circ} \mathrm{C}$. The fused silica capillary column was flushed with $1 \mathrm{~mol} /$ Lsodium hydroxide for $30 \mathrm{~min}$ and deionized water for $10 \mathrm{~min}$ before use. Prior to work, the column was rinsed with $0.1 \mathrm{~mol} / \mathrm{Lsodium}$ hydroxide, deionized water, and working buffer solution, respectively. The capillary was rinsed with $0.1 \mathrm{~mol} / \mathrm{L}$ sodium hydroxide and deionized 
water for two minutes between injections. Then the column was flushed for five minutes with working buffer.

Bioactivity measurements were carried out by a BioTek PowerWave ${ }^{\mathrm{TM}} \mathrm{XS}$ (Winooski, VT, USA) 96-well microplate reader coupled with Gen5 Data Analysis software. The correlations between the oleuropein amounts and the bioactivities of olive leaf extracts were evaluated by Pearson's correlation coefficient.

The solutions were prepared using deionized water obtained from Elga Purelab Option-7-15 water purification system (Elga, UK).

\subsection{DPPH Radical Scavenging Activity Assay}

Olive leaf extracts were analyzed by the method developed by Blois [18].Ten $\mu \mathrm{L}$ of each sample solution were added to $90 \mu \mathrm{L}$ methanolic $0.1 \mathrm{mM}$ DPPH solution. The final concentrations of the extracts in solutions were $10,25,50$, and $100 \mu \mathrm{g} / \mathrm{mL}$. After the extracts waited for thirty minutes in the dark, the absorbances were measured at $515 \mathrm{~nm}$. The percentage inhibition of DPPH free radical was calculated by the following equation:

Percentage inhibition $(\mathrm{I} \%)=\left[\left(\mathrm{A}_{\text {control }}-\mathrm{A}_{\text {sample }}\right) / \mathrm{A}_{\text {control }}\right] \times 100$

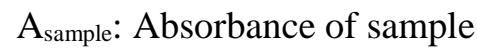

$\mathrm{A}_{\text {control }}$ : Absorbance of control

$\mathrm{IC}_{50}(50 \%$ inhibitory concentration) was calculated by curves constructed from inhibition $\%$ values, and the corresponding sample extract concentrations. The synthetic antioxidant, BHA, was served as a positive control.

\section{$2.5 \alpha$-Glucosidase Enzyme Inhibitory Assay}

Antidiabetic activity is commonly measured by testing the $\alpha$-glucosidase enzyme inhibition power of extracts. The $\alpha$-glucosidase enzyme inhibitory activity is based on the measurement of $p$ nitrophenol releasing from $p$-NPG in the presence of $\alpha$-glucosidase. The method described by Shai et al. was used with a little modification [19]. Fifty $\mu \mathrm{L}$ of phosphate buffer solution $(100 \mathrm{mM})$ at $\mathrm{pH}: 6.8,10$ $\mu \mathrm{L}$ of $\alpha$-glucosidase enzyme $(0.5 \mathrm{U} / \mathrm{mL})$, and $20 \mu \mathrm{L}$ of extracts prepared at $10,25,50,100 \mu \mathrm{g} / \mathrm{mL}$ concentrations were pipetted onto the microplate and waited for $15 \mathrm{~min}$ at $37^{\circ} \mathrm{C}$. Twenty $\mu \mathrm{L}$ of $5 \mathrm{mM} p$ NPG substrate was added for the initiation of an enzymatic reaction. After twenty minutes of incubation, the reaction was ended with the addition of $50 \mu \mathrm{L}$ of $100 \mathrm{mmol} / \mathrm{L}$ sodium carbonate solution. The absorbance of $p$-nitrophenol was monitored at $405 \mathrm{~nm}$ using MicroplateReader. Genistein, which is known as a $\alpha$-glucosidase enzyme inhibitor, was also tested as a positive control. The negative control was the solution without the olive leaf extract. The inhibition percentage of $\alpha$-glucosidase was calculated according to the Eq.1.

\subsection{Anti-inflammatory Activities of Olive Leaf Samples}

Tumor necrosis factor- $\alpha(\mathrm{TNF}-\alpha)$ is a pro-inflammatory cytokine, which production in high levels causes inflammation. The TNF- $\alpha$ inhibition measurement is one of the most common ways for the evaluation theanti-inflammatory activity. The serum levels of TNF- $\alpha$ of sample extracts were determined by in vitro enzyme-linked immunosorbent assay (ELISA) kit based on the protocol obtained by the manufacturer (ThermoFisher Scientific, Waltham, MA). The assay was employed on a 96-well plate coated with an antibody certain to TNF- $\alpha$. The sample extracts were loaded into the wells. The immobilized antibody bounded the TNF- $\alpha$ present in the samples. Each well was washed by washing buffer, and a biotinylated antibody reagent was pipetted into the wells. Before horseradish peroxidase (HRP)-conjugated streptavidin addition, the unbound biotinylated antibody was washed away. The wells 
were washed by washing buffer after an incubation step. Each well was filled with the TMB substrate (3,3',5,5'-tetramethylbenzidine).

According to the amount of bounded TNF- $\alpha$, blue color was formed. Finally, the reaction was finished by adding the stop solution ( $1 \mathrm{M}$ phosphoric acid) and the blue color turned into yellow. MicroplateReader was used to detect the color intensity at $450 \mathrm{~nm}$. Inhibition of TNF- $\alpha$ in percent (I \%) was calculated by using Eq.1.

\section{Results and Discussion}

\subsection{Capillary Electrophoretic Separation of Oleuropein}

Under alkali conditions, polyphenols are mostly negatively charged which ensures the separation depending on charge-to-mass ratios of the deprotonated polyphenols. Borate $(\mathrm{pKa}=9.6)$ is one of the most effective running buffers in the capillary electrophoretic separation of phenolic compounds. In this study, borate was chosen as the separation buffer.

In order to optimize the analysis condition, the running buffer concentration and $\mathrm{pH}$ were firstly considered. The borate concentration was changed between 20 and $40 \mathrm{mM}$ at pH 9.6. $30 \mathrm{mM}$ was chosen as the optimal concentration for oleuropein in terms of peak height, peak symmetry, and migration time. The effect of $\mathrm{pH}$ was examined by adjusting the borate buffer $\mathrm{pH}$ between 9.0 and 10.0. Eventually, it was found that $\mathrm{pH} 9.6$ represented the most satisfactory analysis time.

The separation medium was fixed to $30 \mathrm{mmol} / \mathrm{L}$ borate at $\mathrm{pH}: 9.6$, and an olive leaf extract was injected to CE. However, the separation of oleuropein from minor compounds present in the electrophoretic profile was poor in this buffer system (Figure1a). In order to obtain a good resolution for oleuropein, 2-HP- $\beta$-CD solution was added into the borate in the changing concentration between 10 and $30 \mathrm{mmol} / \mathrm{L}$. The optimum separation was obtained with $25 \mathrm{mmol} / \mathrm{L} 2-\mathrm{HP}-\beta-\mathrm{CD}$ (Figure $1 \mathrm{~b}$ ). We also investigated the effect of methanol. A visible improvement both in the resolution and peak intensity of oleuropein was obtained with the addition of $10 \%(v / v)$ methanol into the buffer as seen from Figure1c. Consequently, we chose $30 \mathrm{mmol} / \mathrm{Lborate}(\mathrm{pH} 9.6), 25 \mathrm{mmol} / \mathrm{L} 2-\mathrm{HP}-\beta-\mathrm{CD}, 10 \%(v / \mathrm{v})$ methanol as the optimal separation medium.

\subsection{Method Validation}

Intra- and interday repeatabilities were performed in order to determine the precision. Standard oleuropein was injected to CE for six times in 1 day and 3 different days. RSD \% values both for the retention times and corrected peak areas were lower than 2.50 and $4.50 \%$ for the intra-day precision and inter-day precision, respectively. The method linearity was checked by plotting peak areas as a function of oleuropein concentration. The calibration graph was obtained by six levels in the range of 50-1000 $\mu \mathrm{g} / \mathrm{mLwith}$ a correlation coefficient of 0.996 . The calibration equation was $\mathrm{y}=0.00036 \mathrm{x}-0.00402$. LOD and LOQ values were calculated as three and ten times of the $\mathrm{S} / \mathrm{N}$, respectively. The LOD value was $13.4 \mu \mathrm{g} / \mathrm{mL}$, and the LOQ was $44.8 \mu \mathrm{g} / \mathrm{mL}$ for oleuropein.

The olive leaf extacts were spiked with standard analyte solution corresponding to 50,100, and $200 \%$ of the oleuropein concentration in the real sample. Thereby, OL1 was fortified with 53, 106, and $212 \mathrm{mg} / \mathrm{g}$ oleuropein. OL7 was spiked with 5.85, 11.7, and $23.4 \mathrm{mg} / \mathrm{g}$ whereas Brand-1 was fortified with $17.9,35.8$, and $71.6 \mathrm{mg} / \mathrm{g}$ oleuropein, respectively. According to CE analysis of spiked samples, the recovery percentage of oleuropein was calculated. Satisfactory recovery values were obtained between $106 \%$ and $109 \%$ (Table S1 in supporting information).

The estimated standard uncertainty, $\mathrm{u}$, was calculated from the repeatability of sample measurements and the method's intra-day precision. The combined uncertainty (U) was found by multiplying u by $\mathrm{k}$, where $\mathrm{k}$ was chosen 2 for $95 \% \mathrm{CL}$. U values are given in Table 1. 


\subsection{Selection of Extraction Condition of Oleuropein from Olive Leaf}

A certain amount of olive leaf was extracted in different solvents at different compositions. In this way, the solvent effect on the extraction efficiency of oleuropein was determined. Each time $0.1 \mathrm{~g}$ of olive leaf was extracted with $5 \mathrm{~mL}$ of five different extraction solvents (water, $100 \%$ methanol, $100 \%$ ethanol, $80 \%$ methanol - 20\% water $(v / v), 80 \%$ ethanol - $20 \%$ water $(v / v))$ for 1 hour by ultrasonicator. After centrifugation and filtration, the residues were re-extracted with their own extraction solvents for $30 \mathrm{~min}$. Combined extracts were completed to $10 \mathrm{~mL}$, filtered through $0.45 \mu \mathrm{m}$ pore-sized microfilters and injected to the capillary.

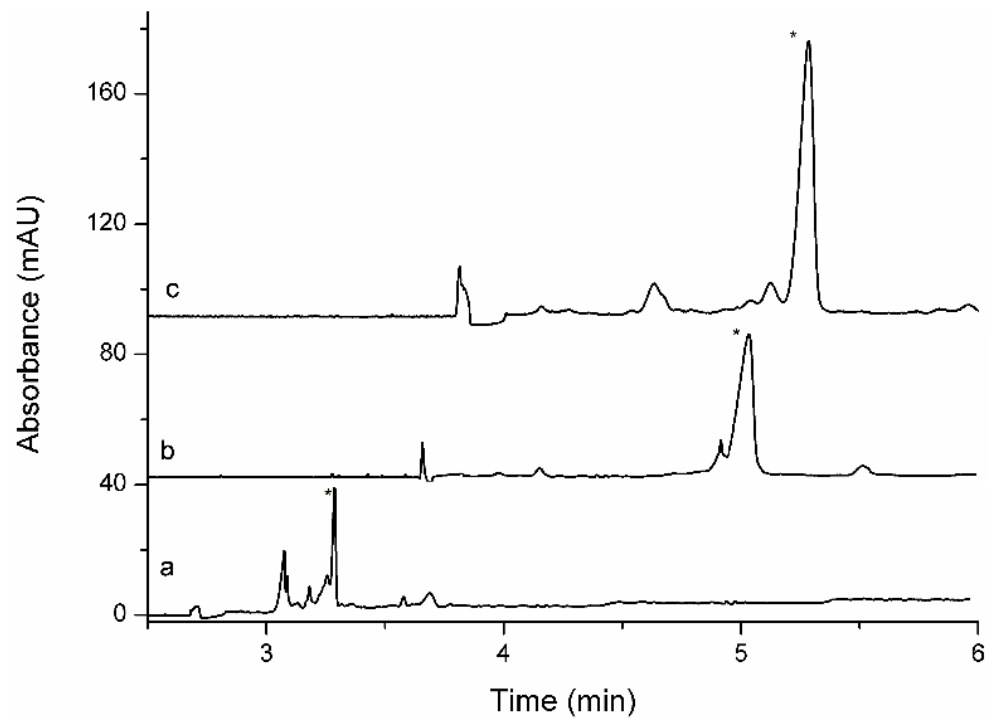

Figure 1.Electropherogram of an olive leaf extract (OL1) in a) $30 \mathrm{mM}$ borate buffer at $\mathrm{pH}$ : 9.6 ; b) $30 \mathrm{mM}$ borate $+25 \mathrm{mM} 2-\mathrm{HP}-\beta-\mathrm{CD}$ at $\mathrm{pH}: 9.6$; c) $30 \mathrm{mM}$ borate $+25 \mathrm{mM} 2-\mathrm{HP}-\beta-\mathrm{CD}+10 \%(v / v)$ methanol at $\mathrm{pH} 9.6 \mathrm{~V}: 25 \mathrm{kV}$, t: $25^{\circ} \mathrm{C}$, Injection: 50 mbar, $6 \mathrm{~s}$.

The peak areas obtained from each extracts were compared. The highest extraction efficiency for oleuropein was obtained when $80 \%$ methanol - $20 \%$ water $(v / v)$ combination and this mixture was used as extraction solvent. The olive leaves were ultrasonicated in the $80 \%$ methanol $-20 \%$ water $(v / v)$ for different times. According to the relative peak areas, the maximum oleuropein amount was obtained in 1 $\mathrm{h}$. Since the peak area did not change after $1 \mathrm{~h}, 1 \mathrm{~h}$ ultrasonication was chosen for each sample.

\subsection{Oleuropein Amount of Olive Leaf Samples}

The optimized extraction and CE methods were applied for the determination of oleuropein in olive leaf samples collected from 7 different locations in Turkey. Two different brands of commercial olive leaf were also analyzed. The olive leaf extract was prepared as described above. Figure1c shows the electrophoregram of an olive leaf extract (collected from Bursa) obtained in the certain conditions. The oleuropein contents of olive leaf samples are represented in Table 1. As seen from Table 1, oleuropein content of olive leaf samples varied between 11.7 (Muğla) and 106 (Bursa) mg/g. Oleuropein amounts in commercial brands (Brand 1 and Brand 2) were inside the limits of the amounts of the collected samples. 
Table 1. Oleuropein contents in olive leave samples

\begin{tabular}{ccc}
\hline Olive leaf sample & Collection Locations & Oleuropein $(\mathbf{m g} / \mathbf{g ~ D W} \pm \mathbf{U})^{*}$ \\
\hline OL1 & Bursa & $106 \pm 6.0$ \\
OL2 & İzmir & $61.3 \pm 3.4$ \\
OL3 & Manisa & $45.5 \pm 2.4$ \\
OL4 & İstanbul & $38.2 \pm 2.6$ \\
OL5 & Balıkesir & $17.9 \pm 0.7$ \\
OL6 & Yalova & $17.2 \pm 0.6$ \\
OL7 & Muğla & $11.7 \pm 0.4$ \\
Brand1 & & $35.8 \pm 3.7$ \\
Brand2 & & $34.7 \pm 4.0$ \\
\hline DW: dry weight & & \\
${ }_{\mathrm{k}=2}$ & &
\end{tabular}

Many factors, such as soil type and its content, may affect the oleuropein content. In olive leaves of 11 French varieties, oleuropein was found in the range of $92.7-134 \mathrm{mg} / \mathrm{g}$ by an HPLC technique [14].Ansari et al. analyzed 8 olive leaf cultivars from Iran by HPLC and found oleuropein between $6.1-$ $13 \mathrm{mg} / \mathrm{g}$ [13]. Oleuropein content of Syrian olive leaves $(\mathrm{n}=7)$ reported as $4.3-9.2 \mathrm{mg} / \mathrm{g}$ [15]. Şahin et al. reported the oleuropein amount of one olive variety from İzmir province as $37.84 \mathrm{mg} / \mathrm{g}$ [20].Can et al. analyzed 14 Turkish olive leaf cultivars and found the average oleuropein as $3.24 \mathrm{mg} / \mathrm{g}$ [21].

\subsection{Antioxidant Activity of Olive Leaf Samples}

The results of DPPH scavenging activities of the tested extracts were reported as $\mathrm{IC}_{50}(\mu \mathrm{g} / \mathrm{mL})$. The DPPH scavenging activities of the samples were between 63.2 and $163 \mu \mathrm{g} / \mathrm{mL}$. The lower $\mathrm{IC}_{50}$ values indicate a higher DPPH scavenging activity. As seen from Table 2, the olive leaf collected from Bursa province $(\mathrm{OL} 1)$ has the highest antioxidant activity $(63.2 \mu \mathrm{g} / \mathrm{mL})$, followed by that of İzmir province (OL2) $(79.0 \mu \mathrm{g} / \mathrm{mL})$. Among 9 samples, the olive leaves collected from the region of İstanbul (OL4) (163 $\mu \mathrm{g} / \mathrm{mL})$ and Muğla (OL7) $(161 \mu \mathrm{g} / \mathrm{mL})$ showed the lowest antioxidant activity.

Our results are consistent with the literature in terms of $\mathrm{IC}_{50}$ value of DPPHradical scavenging assay. $\mathrm{IC}_{50}$ of Pakistan olive leaves $(\mathrm{n}=8)$ were found in the range of $22.46-198 \mu \mathrm{g} / \mathrm{mL}$ [22].

Oleuropein is the substantial antioxidant component in olive leaf. Thus, the correlation between oleuropein content in the sample extracts and the DPPH scavenging activity was searched. As was expected, the correlation between the oleuropein contents and the $\mathrm{IC}_{50}$ values of the olive leaf extracts was negatively strong $(r=-0.833)(p<0.05)$.

\section{6. $\alpha$-Glucosidase Enzyme Inhibition Activity}

The $\alpha$-glucosidase inhibition effects of the olive leaf extracts were given in Table 2. Samples collected from Bursa (OL1) and İzmir (OL2) provinces presented the lowest $\mathrm{IC}_{50}$ values (23.8 and 24.5 $\mu \mathrm{g} / \mathrm{mL}$, respectively), indicating the highest $\alpha$-glucosidase inhibition activities. Sample from Muğla (OL7) was the least active $(106 \mu \mathrm{g} / \mathrm{mL})$. The correlation between oleuropein content and $\mathrm{IC}_{50}$ values was negatively strong $(\mathrm{r}=-0.703)(p<0.05)$. Oleuropein has received much attention for its antidiabetic activity [7]. Thus it can be assumed that there is a strong relationship between oleuropein and the antidiabetic effect of olive leaf.

There are many studies on the antidiabetic effect of olive leaf in the literature. The results of the ex-vivo studies showed that the treatment with olive leaf extract has a beneficial hypoglycemic effect both in diabetic people and rats $[4,23,24]$. According to our literature survey, $\alpha$-glucosidase inhibition activity of olive leaf extract has not been reported before. 


\subsection{Anti-inflammatory Activities of Olive Leaf Samples}

Anti-inflammatory effects of the olive leaf extracts were reported as TNF- $\alpha$ inhibition in percent, as seen in Table 2. The olive leaf samples collected from the Bursa (OL1) (73.4\%) and Manisa (OL3) (71.3\%) provinces inhibited TNF- $\alpha$ almost equally. Brand 1 (63.3\%) and OL2 (62.2\%) showed more or less the same effect on TNF- $\alpha$, as well. TNF- $\alpha$ inhibitory effects of the rest of the samples changed between $48.9 \%$ (Brand 2) and 35.1\% (OL6). Most notably, there is no extract showing anti-inflammatory effect lower than that of low control.

The correlation was tested to link the anti-inflammatory activity measured by the TNF- $\alpha$ inhibition method and the oleuropein contents of olive leaf samples. According to Pearson's correlation coefficient, our study showed a highly positive correlation $(\mathrm{r}=0.780)(p<0.05)$.

It is well-known that oleuropein possesses a high anti-inflammatory effect against proinflammatory agents, including tumor necrosis factor alpha (TNF- $\alpha$ ) [9]. Anti-inflammatory effects of olive leaf extracts were also studied by in-vivo techniques with several rat models $[25,26]$. In the study of Qabaha et al., a significant decrease was observed in TNF- $\alpha$ secretion level in cell cultures treated with olive leaf extract [27]. Our study shows a clear correlation between the oleuropein amount of olive leaf and the anti-inflammatory effects of extracts $(r=0.780)$. There is no study searching the TNF- $\alpha$ inhibition of olive leaf extracts by an in-vivo technique, to the best of our knowledge. Moreover, the antiinflammatory activities of olive leaf extracts from different regions of Turkey were compared in this study for the first time.

Table 2. Biological activities of the olive leaf samples

\begin{tabular}{lccc}
\hline $\begin{array}{l}\text { Olive leaf } \\
\text { sample }\end{array}$ & $\begin{array}{c}\text { DPPH radical } \\
\text { scavenging activity } \\
\left(\mathbf{I C}_{\mathbf{5 0}, \boldsymbol{\mu g} / \mathbf{m L})}\right.\end{array}$ & $\begin{array}{c}\boldsymbol{\alpha} \text {-Glucosidase } \\
\text { enzyme inhibitory } \\
\text { activity (IC } \mathbf{5 0}, \boldsymbol{\mu g} / \mathbf{m L})\end{array}$ & $\begin{array}{c}\text { TNF- } \boldsymbol{\alpha} \text { inhibition } \\
(\boldsymbol{\%})\end{array}$ \\
\hline OL1 & $63.2 \pm 1.2$ & $23.8 \pm 1.4$ & $73.4 \pm 1.1$ \\
OL2 & $79.0 \pm 0.9$ & $24.5 \pm 2.6$ & $62.2 \pm 2.7$ \\
OL3 & $102 \pm 2$ & $36.4 \pm 2.8$ & $71.3 \pm 4.3$ \\
OL4 & $163 \pm 3$ & $45.9 \pm 3.1$ & $44.1 \pm 3.7$ \\
OL5 & $145 \pm 5$ & $87.9 \pm 3.9$ & $45.2 \pm 3.7$ \\
OL6 & $130 \pm 4$ & $83.7 \pm 5.4$ & $35.1 \pm 1.1$ \\
OL7 & $161 \pm 2$ & $106 \pm 6$ & $43.6 \pm 1.1$ \\
Brand1 & $152 \pm 3$ & $43.7 \pm 3.4$ & $63.3 \pm 2.7$ \\
Brand2 & $133 \pm 4$ & $70.6 \pm 4.8$ & $48.9 \pm 4.3$ \\
BHA & $7.02 \pm 0.05$ & $4.16 \pm 0.18$ & \\
Genistein & & & $96.3 \pm 4.8$ \\
Control high & & & $31.4 \pm 3.7$ \\
Control low & & & \\
\hline
\end{tabular}

\section{Conclusions}

In this study, the capillary electrophoresis technique developed for the oleuropein was applied to nine different Anatolian olive leaves, two of which were commercially available. In fact, oleuropein contents show quantitative differences, which are related to the variety of the olive leaves. Since the developed method provides good repeatability and accuracy, it can be used as an analytical tool to authenticate olive leaves and to determine their quality. Additionally, we have demonstrated the relation between antioxidant, antidiabetic, and anti-inflammatory activities of extracts and their oleuropein contents. To conclude, olive leaf, as an inexpensive and rich source of oleuropein, could be useful for the improvement of new medicines in the battle of diabetes and inflammation. 


\section{Acknowledgments}

This work was supported by the Research Foundation of Istanbul Technical University. Capillary electrophoretic studies are part of the master thesis of Merve Kopar.

\section{Supporting Information}

Supporting information accompanies this paper on http://www.acgpubs.org/journal/ journalof-chemical-metrology

\section{ORCID}

ZeynepKalaycioğlu: 0000-0002-0967-0997

MerveKopar:0000-0003-2100-3844

F. BediaErim: 0000-0001-9406-6681

\section{References}

[1] M.A. Hashmi, A. Khan, M. Hanif, U. Farooq and S. Perveen (2015). Traditional uses, phytochemistry, and pharmacology of Olea europaea (Olive), Evid. Based. Complement. Alternat. Med. Article ID 541591129.

[2] The Turkish olive oil sector, International Olive Council, Olivae, 123, (2016) URL http://www.olioofficina.net-/knowledge/economy/the-turkish-olive-oil-sector.htm. Accessed: 28.08.2018.

[3] S. Şahin and M. Bilgin (2017). Olive tree (Olea europaea L.) leaf as a waste by-product of table olive and olive oil industry: a review, J. Sci. Food Agric 98, 1271-1279.

[4] A. Kısa, M. Akyüz, H. Y. Çoğun, Ş. Kordali, A. Usanmaz Bozhüyük, B. Tezel, U. Şiltelioğlu, B. Anıl and A. Çakır (2018).Effects of Olea europaea L. leaf metabolites on the Tilapia (Oreochromis niloticus) and three stored pests, Sitophilus granarius, Tribolium confusum and Acanthoscelides obtectus, Rec. Nat. Prod. 12, 201-215.

[5] M.M. Özcan and B. Matthäus (2017). A review: benefit and bioactive properties of olive (Olea europaea L.) leaves, Eur. Food Res. Technol. 243, 89-99.

[6] H.P. Fleming, W.M. Jr. Walter and J.L. Etchells (1973). Antimicrobial properties of oleuropein and products of its hydrolysis from green olives, J. Appl. Microbiol. Biochem. 26, 777-782.

[7] F. Hadrich, Z. Bouallagui, H. Junkyu, H. Isoda and S. Sayadi (2015). The $\alpha$-glucosidase and $\alpha$-amylase enzyme inhibitory of hydroxytyrosol and oleuropein, J. Oleo Sci. 64, 835-843.

[8] H. Jemai, A. El Feki and S. Sayadi (2009). Antidiabetic and antioxidant effects of hydroxytyrosol and oleuropein from olive leaves in alloxan-diabetic rats, J. Agric. Food Chem. 14, 8798-8804.

[9] A.R. Khalatbary and GhR. Zarrinjoei (2012). Anti-inflammatory effect of oleuropein in experimental rat spinal cord trauma, Iran Red. Crescent.Med. J.14, 229-234.

[10] M. Seçme, C. Eroğlu, Y. Dodurga and G. Bağc1 (2016). Investigation of anticancer mechanism of oleuropein via cell cycle and apoptotic pathways in SH-SY5Y neuroblastoma cells, Gene 585, 93-99.

[11] B. Barbaro, G. Toietta, R. Maggio, M. Arciello, M. Tarocchi, A. Galli and C. Balsano (2014). Effects of the olive-derived polyphenol oleuropein on human health, Int. J. Mol. Sci. 15, 18508-18524.

[12] Ö.F. Gamlı (2016). The health effects of oleuropein, one of the major phenolic compounds of olives, Olea europeaL., Ital. J. Food Sci. 28, 178-189.

[13] M. Ansari, M. Kazemipour and S. Fathi (2011). Development of a simple green extraction procedure and HPLC method for determination of oleuropein in olive leaf extract applied to a multi-source comparative study, J. Iran. Chem. Soc. 8, 38-47.

[14] C. Savournin, B. Baghdikian, R. Elias, F. Dargouth-Kesraoui, K. Boukef and G. Balansard (2001). Rapid high-performance liquid chromatography analysis for thequantitative determination of oleuropein in Olea europaea leaves, J. Agric. Food Chem. 49, 618-621.

[15] G. Tayoub, H. Sulaiman, A.H. Hassan and M. Alorfi (2012). Determination of oleuropein in leaves and fruits of some Syrian olive varieties, Int. J. Med. Aromat. Plants 2, 428-433. 
[16] C.A. Ballus, A.D. Meinhart, R.E. Bruns and H.T. Godoy (2011). Use of multivariate statistical techniques to optimize the simultaneous separation of 13 phenolic compounds from extra-virgin olive oil by capillary electrophoresis, Talanta 83, 1181-1187.

[17] M. Bonoli, M. Montanucci, T.G. Toschi and G. Lercker (2003). Fast separation and determination of tyrosol, hydroxytyrosol and other phenolic compounds in extra-virgin olive oil by capillary zone electrophoresis with ultraviolet-diode array detection, J. Chromatogr. A 1011, 163-172.

[18] M. S. Blois (1958). Antioxidant determinations by the use of a stable free radical, Nature18, 1199-1200.

[19] L.J. Shai, S.R. Magano, S.L. Lebelo and A.M. Mogale (2011). Inhibitory effects of five medicinal plants on rat alpha-glucosidase: comparison with their effects on yeast alpha-glucosidase, J. Med. Plant Res.5, 2863-2867.

[20] S. Şahin, M. Bilgin and M.U. Dramur (2011). Investigation of oleuropein content in olive leaf extract obtained by supercritical fluid extraction and soxhlet methods, Sep. Sci. Technol. 46, 1829-1837.

[21] A. Can, H. Ayvaz, Ç. Uysal Pala, N. Condelli, F. Galgano and R. Tolve (2018). The potential of near and mid-infrared spectroscopy for rapid quantification of oleuropein, total phenolics, total flavonoids and antioxidant activity in olive tree (Olea europaea) leaves, J. Food Meas. Charact. 12, 2747-2757.

[22] A. Khaliq, S.M. Sabir, S.D. Ahmad, A.A. Boligon, M.L. Athayde, A. Jabbar, I. Qamar and A. Khan (2015). Antioxidant activities and phenolic composition of Olive (Olea europaea) leaves, J. Appl. Bot. Food Qual. 88, 16-21.

[23] A. Eidi, M. Eidi and R. Darzi (2009). Antidiabetic effect of Olea europaeaL. in normal and diabetic rats, Phytother. Res. 23, 347-350.

[24] J. Wainstein, T. Ganz, M. Boaz, Y.B. Dayan, E. Dolev, Z. Kerem and Z. Madar (2012). Olive leaf extract as a hypoglycemic agent in both human diabetic subjects and in rats, J. Med.Food 7 , 1-6.

[25] E. Haloui, B. Marzouk, Z. Marzouk, A. Bouraoui and N. Fenina (2011). Hydroxytyrosol and oleuropein from olive leaves: Potent anti-inflammatory and analgesic activities,J. Food Agric. Environ. 9, 128-133.

[26] T. Vezza, F. Algieri, A. Rodriguez-Nogales, J. Garrido-Mesa, M.P. Utrilla, N. Talhaoui, A.M. GomezCaravaca, A. Segura-Carretero, M.E. Rodriguez-Cabezas, G. Monteleone and J. Galvez (2017). Immunomodulatory properties of Olea europaea leaf extract in intestinal inflammation, Mol. Nutr. Food Res. 61, 1-9.

[27] K. Qabaha, F. Al-Rimawi, A. Qasem and S.A. Naser (2018). Oleuropein is responsible for the major antiinflammatory effects of olive leaf extract, J. Med. Food 21, 302-305.

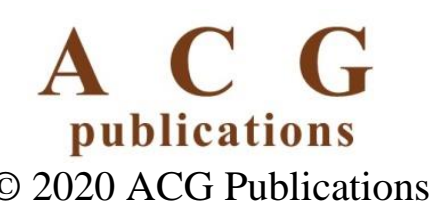

